\title{
Wavelet Chaotic Neural Network with Function Disturbance
}

\author{
Yaoqun $X U^{1, a}$, Jian LIU ${ }^{2, b}$ \\ ${ }^{1}$ School of Science, Harbin University of Commerce, Harbin, 150028, China \\ ${ }^{2}$ Department of Mathematic, Harbin University of Commerce, Harbin, 150028, China \\ aemail: xuyq0630@163.com, bemail:hrbcumath@163.com
}

Keywords: Chaotic Neural Network, Function Disturbance, Traveling salesman problem

\begin{abstract}
Chaotic neural networks have been proved to be strong tools to solve the optimization problems. In order to study the anti-disturbance ability of chaotic neural network, wavelet function disturbance was introduced into morlet wavelet chaotic neural network and chaotic neural units with wavelet function disturbance were studied. The reversed bifurcation and the maximum Lyapunov exponent were given and the dynamic system was analyzed. The simulation results show that the chaotic neural network with function disturbance can solve function and combinatorial optimization problems effectively, if the disturbance coefficient is controlled properly. Therefore the very strong robustness and anti-interference ability are embodied in chaotic neural network.
\end{abstract}

\section{Introduction}

Some developments in nonlinear dynamics and the theory of chaos have shown deterministic chaotic property of EEGs. Such research made the researchers try to utilize the chaotic behavior in artificial neural networks. Chaotic Neural networks (CNNs) have been shown to be powerful tools for solving optimization problems, particularly NP-hard problems. Chaotic neural networks (CNNs) can acquire the ability to escape local minima of energy functions by chaotic dynamics [1-11]. Several chaotic neural networks with non-monotonous activation functions have been proved to be more powerful than Chen's chaotic neural network in solving optimization problems, especially in searching global minima of continuous function and traveling salesman problems. Chen and Aihara have proposed a CNN with CSA by introducing a linear self-feedback into HNN and reducing the self-feedback connection weight exponentially which ensures that has transient chaotic search behavior and can converge to a point steadily [1]. Chen and Aihara prove that the proposed model is asymptotical stability. Yang proposed a delayed chaotic neural network with annealing controlling strategies to solve the NP-complete maximum clique problem (MCP)[2]. Sun et al. studied a novel hysteretic noisy chaotic neural network for broadcast scheduling problems in packet radio networks and exhibited a stochastic chaotic simulated annealing algorithm [6].

In order to study the anti-disturbance of chaotic neural network, the disturbance was introduced into chaotic neural network and chaotic neural units with wavelet function disturbance were studied. The reversed bifurcation and the maximum Lyapunov exponent were given and the dynamic system was analyzed. It is applied to the function optimization problems and combinatorial optimization problem. Some simulations show that the wavelet chaotic neural network with disturbance can utilize chaotic dynamics to search, and settle the function optimization problems and combinatorial optimization problem effectively, if disturbance coefficient is controlled properly. Therefore the very strong robustness and anti-interference ability are embodied in chaotic neural network.

\section{Wavelet Chaotic Neural Network with Function Disturbance}

A wavelet is a wave-like oscillation with an amplitude that begins at zero, increases, and then decreases back to zero. Wavelet chaotic neural network is a very complicate nonlinear system, because wavelet function is a kind of basic function, the model can solve optimization problems more effectively. A novel chaotic neural network model can be described as follows: 


$$
\begin{aligned}
& y_{i}(t+1)=k y_{i}(t)+\gamma M\left(y_{i}(t), \varepsilon\right)+\alpha\left[\sum w_{i j} x_{j}+I_{i}\right]-z_{i}(t)\left(x_{i}(t)-I_{0}\right) \\
& x_{i}(t+1)=S\left(y_{i}(t+1) / \varepsilon_{1}\right)+c M\left(y_{i}(t+1), \varepsilon_{2}\right) \\
& z_{i}(t+1)=(1-\beta) z_{i}(t) \\
& M\left(y_{i}(t), s\right)=\exp \left(-\left(y_{i}(t) / s\right)^{2} / 2\right) \cos \left(5 y_{i}(t) / s\right)
\end{aligned}
$$

\begin{tabular}{|c|c|}
\hline symbol & meaning \\
\hline$x_{i}$ & the output of neuron \\
\hline$y_{i}$ & the internal state of neuron $i$ \\
\hline$w_{i j}$ & the connection weight, $w_{i j}=w_{j i}$ \\
\hline$I_{i}$ & input bias of neuron $i$ \\
\hline$I_{0}$ & positive parameter \\
\hline$k$ & damping factor of nerve membrane $(0<k<1)$ \\
\hline$\varepsilon, \varepsilon_{1}, \varepsilon_{2}$ & $\begin{array}{l}\text { steepness parameter of the activation function } \\
(\varepsilon>0)\end{array}$ \\
\hline$z_{i}$ & the self-feedback connection weight \\
\hline$\beta$ & the damping factor \\
\hline$c, \gamma$ & the parameter of the nonlinear function \\
\hline
\end{tabular}

where activation function of neurons in the formula (2) is composed of Sigmoid function and Morlet wavelet function. The symbol meaning is Table 1.

Table 1. Symbol meaning

In this model, the variable $z_{i}(t)$ corresponds to the temperature in the usual stochastic annealing process and the equation (3) is an exponential cooling schedule for the annealing. The chaotic mechanism is introduced by the self-feedback connection weight as the value of $z_{i}(t)$ becomes small step by step. The chaotic behavior plays a global search role in the beginning.

We make some analysis of the neural unit of the chaotic neural networks.

The parameters of this neural unit model are set as follows:

$$
\varepsilon_{1}=0.04, \quad \varepsilon_{2}=2.5, \quad \varepsilon=1.25, k=1, \beta=0.001, \gamma=0.002, I_{0}=0.65, \quad y(0)=0.283 \text {, }
$$
$z(0)=0.8, \quad c=1 / 3$.

The bifurcation behavior and the time evolution figures of the maximal Lyapunov exponent are respectively shown as Fig.1(a),(b).

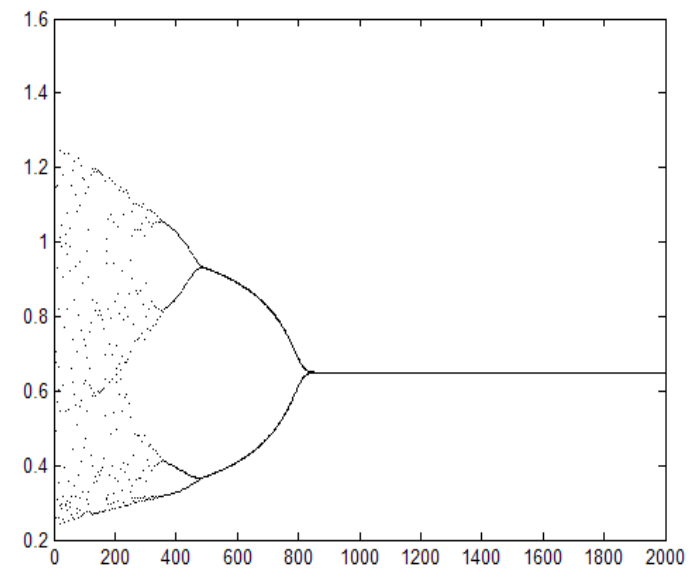

(a) The bifurcation behavior of the neuron

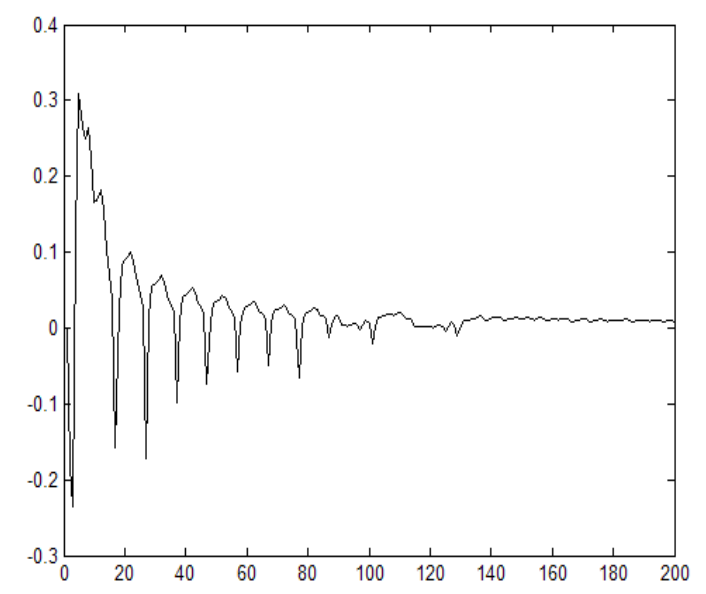

(b)Lyapunov exponent time evolution figure

Fig.1. The experimental results 
Seen from the above the bifurcation behavior, the neuron behaves a transient chaotic dynamic behavior when Lyapunov exponent is above zero. The single neural unit first behaves the global chaotic search, and with the decrease of the value of $z(0,0)$, the reversed bifurcation gradually converges to a stable equilibrium state. After the chaotic dynamic behavior disappears, the dynamic behavior of the single neural unit is controlled by the gradient descent dynamics. When the behavior of the single neural unit is similar to that of Hopfield, the network tends to converge to a stable equilibrium point.

\section{Test results}

The dynamic programming is a branch that it is multi-stage decision-making process of solving a mathematical optimization method. In many research fields, mathematical optimization is the selection of a best element (with regard to some criteria) from some set of available alternatives. We consider the function described as follows:

$$
f\left(x_{1}, x_{2}\right)=\left(x_{1}-0.7\right)^{2}\left[\left(x_{2}+0.6\right)^{2}+0.1\right]+\left(x_{2}-0.5\right)^{2}\left[\left(x_{1}+0.4\right)^{2}+0.15\right]
$$

Zero is the minimum value of the function above at point $(0.7,0.5)$.

We set the parameters as follows:

$$
\mathcal{E}=0.2, k=1, c=1,{ }^{y_{1}(1)}=y_{2}(1)=0.283,{ }^{z_{1}(1)}={ }_{2}{ }_{2}(1)=0.8, \beta=0.003, \alpha=0.5,{ }{ }=0.5
$$

According to simulation, We can get the optimal solution of ${ }_{1}, X_{2}$ (see Fig.2(a)) and the time evolution figure of the energy function(see Fig.2(b)) .
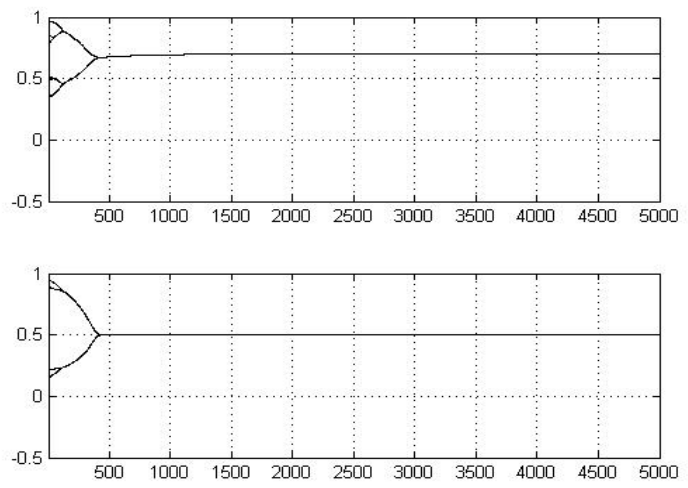

(a) The optimal solution of $X_{1}, X_{2}$

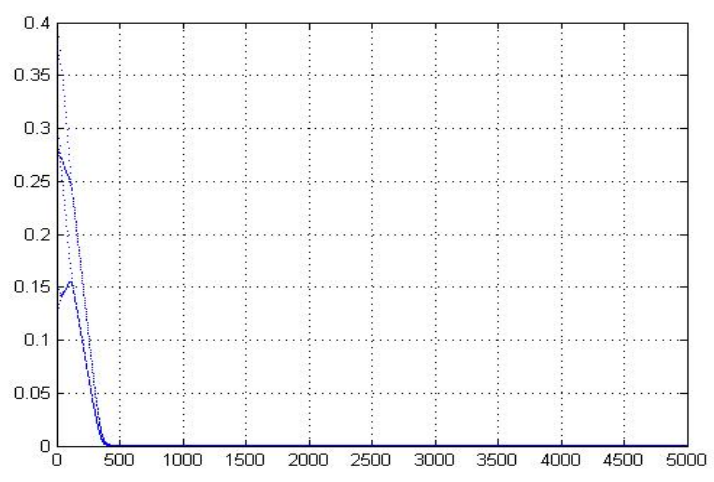

(b) Time evolution figure of energy function

Fig.2. The experimental results

In order to testify the performance of the novel neural network, the proposed neural network is applied to the travelling salesman problem(TSP). The TSP has several applications even in its purest formulation, such as planning, logistics, and the manufacture of microchips. The TSP asks the following question: Given a list of cities and the distances between each pair of cities, what is the shortest possible route that visits each city exactly once and returns to the origin city? It is an NP-hard problem in combinatorial optimization, important in operations research and theoretical computer science. A solution of TSP with $N$ cities is represented by $N \times N$-permutation matrix. In order to solve TSP, we assume ${ }^{X_{i j}}$ to be the neuron output that represents city $i$ in visiting order ${ }^{j}$.

In this paper, we adopt energy function:

$$
E=\frac{W_{1}}{2}\left\{\sum_{i=1}^{n}\left[\sum_{j=1}^{n} x_{i j}-1\right]^{2}+\sum_{j=1}^{n}\left[\sum_{i=1}^{n} x_{i j}-1\right]^{2}\right\}+\frac{W_{2}}{2} \sum_{i=1}^{n} \sum_{j=1}^{n} \sum_{k=1}^{n}\left(x_{k, j+1}+x_{k, j-1}\right) x_{i j} d_{i k}
$$

Where $x_{i 0}=x_{i n}$ and $x_{i, n+1}=x_{i 1} . W_{1}$ and $W_{2}$ are the coupling parameters corresponding to the constraints and the cost function of the tour length, respectively. ${ }^{d x y}$ is the distance between city ${ }^{x}$ and city $^{y}$. 
This paper adopts the following 10-city unitary coordinates:

Table 2. City Coordinates for The Hopfield-Tank Ten-City Problem

\begin{tabular}{cccccc}
\hline \hline$i$ & 1 & 2 & 3 & 4 & 5 \\
\hline \multirow{2}{*}{$X(i)$} & 0.4 & 0.243 & 0.170 & 0.229 & 0.517 \\
& & 9 & 7 & 3 & 1 \\
\hline \multirow{2}{*}{$Y(i)$} & 0.443 & 0.146 & 0.229 & 0.716 & 0.941 \\
& 9 & 3 & 3 & 4 & 4 \\
\hline \multirow{2}{*}{$X$} & 6 & 7 & 8 & 9 & 10 \\
\hline \multirow{2}{*}{$X(i)$} & 0.873 & 0.687 & 0.848 & 0.668 & 0.619 \\
& 2 & 8 & 8 & 3 & 5 \\
\hline \multirow{2}{*}{$Y(i)$} & 0.653 & 0.521 & 0.360 & 0.253 & 0.263 \\
& 6 & 9 & 9 & 6 & 4 \\
\hline \hline
\end{tabular}

The shortest distance of the 10 -city is 2.6776 .

The parameters and energy coefficients are very important factors related to the efficiency of neural networks. We initialize the parameters as follow:

$$
\begin{gathered}
W_{1}=1, \quad W_{2}=0.8, \quad z(0)=0.2, \alpha=0.6, k=1, \quad I_{0}=0.5, \quad \varepsilon_{1}=0.004, \quad \varepsilon_{2}=1.25, \\
\varepsilon=1.25, \quad \beta=0.0004, \quad c=1 / 7
\end{gathered}
$$

One hundred kinds of different initial conditions of yi are generated randomly in the region $[0,1]$ for different $\beta$. The results are summarized in Table2. The column 'NL', 'NG', 'LR' and 'GR' respectively represents the number of legal route, the number of global optimal route, the rate of legal route, the rate of global optimal route.

Table 3. Results of 100 different initial conditions for each value on 10-city TSP

\begin{tabular}{l|r|r|r|l}
\hline MODEL & \multicolumn{1}{|c|}{$\mathrm{N}$} & \multicolumn{1}{|c|}{$\mathrm{N}$} & \multicolumn{1}{l}{ LR } & GR \\
\hline$\gamma=0.2$ & 87 & 17 & $87 \%$ & $17 \%$ \\
\hline$\gamma=0.02$ & 98 & 47 & $98 \%$ & $47 \%$ \\
\hline$\gamma=0.002$ & 97 & 91 & $97 \%$ & $91 \%$ \\
\hline
\end{tabular}

Seen from table 2, when Morlet wavelet neural network with the function disturbance is used to solve 10 city traveling salesman problem by controlling the disturbance coefficient properly such as $\gamma$ is 0.02 , the average success rate of legitimate solution and optimal solution can reach over $97 \%$. When the disturbance coefficient increases such as $\gamma$ is 0.08 or larger, the average success rate of optimal solution and legal solution drop significantly, and even a legal solution or the optimal solution is not found, and the speed of convergence slows down significantly.

The time evolution figure of the energy function of this model in solving TSP is given in Fig.3 


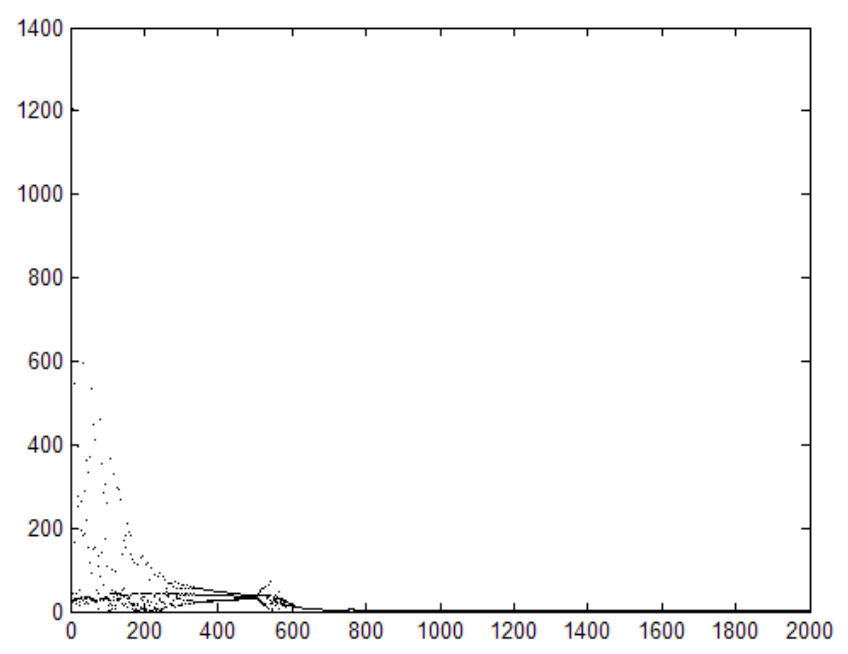

Fig.3. Energy time evolution figure

This model is used for solving the 10 city traveling salesman problem. The parameter $\gamma$ is changed but other parameters are the same as above. The simulation results are shown in Table3.

Table 4. Results of 100 different initial conditions on 10-city TSP

\begin{tabular}{l|l|l|l|l}
\hline MODEL & NL & NG & LR & GR \\
\hline$\gamma=0$ & 97 & 93 & $97 \%$ & $93 \%$ \\
\hline$\gamma=0, c=0$ & 94 & 92 & $94 \%$ & $92 \%$ \\
\hline
\end{tabular}

By a comparison of Table 2 to Table 3, when $\gamma$ is equal to zero, this network model is the wavelet chaotic neural network model. At this time, its performance is best, and the average success rate of legitimate solution and optimal solution can reach $100 \%$. When $\quad \gamma$ and $C$ are all equal to zero , the network is Chen's chaotic neural network model.

From the simulation results above, by controlling the disturbance coefficient properly Morlet wavelet chaotic neural network with the wavelet function disturbance can solve the 10 city traveling salesman problem effectively. It is illustrated fully that Morlet wavelet chaotic neural network model has a strong anti-Interference ability.

\section{Conclusion}

A novel wavelet chaotic neural network model is presented in this paper by introducing the wavelet function disturbance. It can take full advantage of the dynamics properties of chaotic search and solve the optimization problems effectively. It is shown that this chaotic neural network model has a strong anti-interference ability and robustness. According to our research results, this wavelet chaotic neural network mode has a great potential, and lays the foundation for controlling interference in practical applications. With further research, the network model should be applied to other optimization problems.

\section{Acknowledgement}

In this paper, the research was sponsored by The National Soft Science Research Program (Project No. 2013GXS4D115) and the Nature Science Foundation of Heilongjiang Province (Project No. F201035).

\section{References}

[1] L.N. Chen, K. Aihara, Chaotic Simulated Annealing by a Neural Network Model with Transient Chaos[J], Neural Networks, 1995. 8(6): 915-930

[2] Gang Yang, JunyanYi, Delayed chaotic neural network with annealing controlling for maximum 
clique problem[J], Neurocomputing, 2014, 127:114-123

[3] Y. Xu, T. Zhao, Chaotic Neural Network with Nonlinear Function Self-feedback, Proceedings of the 33th Chinese Control Conference CCC2014,Nanjing,2014,5075-5079

[4] Shyan-Shiou Chen, Chaotic Simulated Annealing by a Neural Network with a Variable Delay: Design and Application[J], IEEE Trans. on Neural Networks,,2011.22(10): 1557-1565

[5] Y. Xu, M. Sun, and J. Zhang. A model of wavelet chaotic neural network with applications in optimization, Proceedings of 6th World Congress Intelligent Control Automatic, China,,2006.1: 2901-2905

[6] M. Sun, Y. Xu, X. Dai, Y. Guo. Noise-tuning-based hysteretic noisy chaotic neural network for broadcast scheduling problem in wireless multihop networks[J], IEEE Transactions on Neural Networks and Learning Systems,, 2012.23(12): 1905-1918

[7] L. Chen, K. Aihara. Chaos and asymptotical stability in discrete-time neural networks[J]. Physic D, 104(3-4): 286-325, 1997.

[8] Jun Chen, Jose Suarez, Peter Molnar and Aman Behal, Maximum Likelihood Parameter Estimation in a Stochastic Resonate-and-Fire Neuronal Model, 2011 IEEE International Conference on Computational Advances in Bio and medical Sciences (ICCABS), Orlando, FL, Feb 3-5, 2011.

[9] L. Zhao, M. Sun, J. Cheng, Y. Xu. A novel chaotic neural network with the ability to characterize local features and its application[J]. IEEE Trans. on Neural Networks, 20(4): 737-738, 2009.

[10] Y. Xu, X. Yang. Chaotic neural networks with sigmoid function self-feedback and its applications. ICNC2010, 2010: 1605-1609.

[11] Lingfei Zhi, Jun Chen, Peter Molnar and Aman Behal, Weighted Least-Squares Approach for Identification of a Reduced-Order Adaptive Neuronal Model[J], IEEE Transactions on Neural Networks and Learning Systems, May 2012. 23(5):834-840 